\title{
A BAIRE SPACE EXTENSION
}

\author{
R. A. MCCOY
}

ABSTRACT. A characterization of Baire spaces is given. Using this characterization, it is shown that every topological space is a dense subspace of some compact Baire space.

If $X$ and $Y$ are topological spaces and if $X$ is a dense subspace of $Y$, then $Y$ will be called an extension of $X$. If $Y$ has property $\mathrm{P}$, then it will be called a $\mathrm{P}$ extension of $X$. Many people have investigated, for various non'tereditary properties $P$, the classes of spaces having $P$ extensions. For example, every Tychonoff space has a compact, Hausdorf extension, and every Hausdorff space has an $H$-closed extension [3]. An $H$-closed (or absolutely closed) space is a Hausdorff space such that every open filter base has a cluster point. Since a compact Hausdorff space is a Baire space, then every Tychonoff space has a Tychonoff, Baire space extension. A Baire space is a space such that every nonempty open subset is of second category in the space. Herrlich gave an example in [2] of an $H$-closed space (in fact a minimal Hausdorff space) which is of first category in itself and hence not a Baire space. Therefore there exist Hausdorff spaces whicn have no Hausdorff Baire space extensions. In the Corollary to Theorem 3, we see that every topological space has a compact Baire space extension.

We shall use the following characterization of Baire spaces, which is similar to a characterization given in [1] of spaces of second category in themselves.

THEOREM 1. $X$ is a Baire space if and only if every countable point finite open cover of $X$ is locally finite at a dense sei of points.

Proof. Suppose that $X$ is a Baire space. Let $\mathscr{U}=\left\{U_{i}\right\}$ be a countable point finite open cover of $X$, and let $U$ be a nonempty open subset of $X$. Then $U$ is of second category in $X$. Assume that $\mathscr{U}$ is not locally finite at any point of $U$. Let $\mathscr{V}=\left\{V_{i}\right\}$, where each $V_{i}=U_{i} \cap U$. Then each open set in $U$ intersects infiniteiy many members of $\mathscr{Y}$. Let $\omega$ be the set of natural numbers, and let $\mathscr{N}=\{N \mid N \subset \omega$ and $\omega \backslash N$ is finite $\}$, which is countable. Let $\left\{N_{i} \mid i \in \omega\right\}$ be a well ordering of $\mathscr{N}$. For each $i$, define $X_{i}=$ $\operatorname{Bd}\left[\bigcup\left\{V_{j} \mid j \in N_{i}\right\}\right]$. Each $X_{i}$ is closed and Int $X_{i}=\varnothing$, so that each $X_{i}$ is

Presented to the Society, September 3, 1971; received by the editors April 15, 1971. AMS 1970 subject classifications. Primary 54C25, 54D25, 54D30, 54D35.

Key words and phrases. Baire space, $H$-closed space, open filter, open ultrafilter. 
nowhere dense. Let $x \in U$. There exists an integer $k$ such that $x$ is in the members of $\left\{V_{i} \mid i \in(1) \backslash N_{k}\right\}$, but not in the other members of $\mathscr{V}^{\circ}$. Let $V$ be an open set containing $x$. Then $V$ intersects some members of $\left\{V_{j} \mid j \in N_{k}\right\}$. But since $x \notin \bigcup\left\{V_{j} \mid j \in N_{k}\right\}$, then $x \in X_{k}$. This says that $U=\bigcup_{i=1}^{\gamma_{i}} U \cap X_{i}$, which contradicts $U$ being of second category in $X$.

Conversely, suppose that every countable point finite open cover of $X$ is locally finite at a dense set of points. Let $U$ be a nonempty open subset of $X$. Assume that $U$ is of first category in $X$. Then $U=\bigcup_{i=1}^{\infty} X_{i}$, where Int $\bar{X}_{i}=\varnothing$ for each $i$. Let $U_{0}=X$, and for each $i$, let $U_{i}=U \backslash \bigcup_{j=1}^{i} \bar{X}_{j}$. Let $\mathscr{U}=\left\{U_{0}, U_{1}, \cdots\right\}$, which is a countable point finite open cover of $X$. Then there exists an $x \in U$ such that $\|$ is locally finite at $x$. So there is an open set $V$ such that $x \in V \subset U$ and $V$ intersects only finitely many members of $\pi$. But $V$ is not contained in $\bigcup_{j=1}^{i} \bar{X}_{j}$ for each $i$. Then $V$ must intersect every member of $\%$, which is a contradiction.

Lemma. Let De a base for the topological space $X$. If $X$ is not a Baire space, then there exist a countable point finite collection $\left\{U_{i}\right\}$ of open subsets of $X$ and a countable collection $\left\{B_{i}\right\} \subset \mathscr{B}$ such that $B_{k+1} \subset B_{i i} \cap\left(\bigcap_{i=1}^{k} U_{i}\right)$ for every $k$.

Proof. By Theorem 1, there exist a countable point finite open cover $\mathscr{V}=\left\{V_{i}\right\}$ of $X$ and an open subset $V$ of $X$ such that $\mathscr{V}$ is not locally finite at any point of $V$. Let $B_{1} \in \mathcal{B}$ be contained in $V$. There exists an integer $i_{1}$ such that $B_{1} \cap V_{i_{1}} \neq \varnothing$. Let $B_{2} \in \mathscr{B}$ be contained in $B_{1} \cap V_{i_{1}}$. Proceeding by induction, suppose we have defined distinct $\left\{i_{1}, \cdots, i_{n-1}\right\}$ and $\left\{B_{1}, \cdots, B_{n}\right\} \subset$ $\mathscr{B}$ such that $B_{k} \subset B_{k-1} \cap\left(\bigcap_{j=1}^{k-1} V_{i}\right)$ for every $2 \leqq k \leqq n$. Then since $\mathscr{Y}^{-}$is not locally finite in $V$, there exists an integer $i_{n}$ distinct from the elements of $\left\{i_{1}, \cdots, i_{n-1}\right\}$ such that $B_{n} \cap V_{i_{n}} \neq \varnothing$. Let $\left.B_{n+1} \in \not\right\rangle$ be contained in $B_{n} \cap V_{i_{n}}$. Thus $B_{n+1} \subset B_{n} \cap\left(\bigcap_{j=1}^{n} V_{i}\right)$, so that $\left\{E_{i}\right\}$ is therefore defined by induction. For each $j$, take $U_{j}=V_{i j}$. Then $\left\{U_{i}\right\}$ and $\left\{B_{i}\right\}$ satisfy the conclusion of the Lemma.

An open filter $\tilde{\mathcal{H}}$ on a space $X$ is a nonempty collection of nonempty open subsets of $X$ satisfying:

(a) If $U, V \in \mathscr{F}$, then $U \cap V \in \mathscr{F}$.

(b) If $U \in \tilde{F}$ and $V$ is an open set containing $U$, then $V \in \mathscr{F}$. An open ultrafilter is an open filter which is maximal in the collection of open nilters. An open filter $\mathscr{F}$ is free if $\cap \mathscr{F}=\not \supset$.

THEOREM 2. If $X$ is not a Baire space, then $X$ has a free open ultrafilter.

Proof. Let $\left\{U_{i}\right\}$ and $\left\{B_{i}\right\}$ be defined as in the Lemma. Then $\left\{B_{i}\right\}$ is an open filter base on $X$. Let $\mathcal{U}$ be an open ulirafilter on $X$ containing $\left\{B_{i}\right\}$. Since $\bigcap_{i=1}^{\infty} B_{i} \subset \bigcap_{i=1}^{\infty} U_{i}$ and $\left\{U_{i}\right\}$ is point finite, then $\bigcap_{i=1}^{\infty} B_{i}=\not 2$. Therefore $\cap \|=\varnothing$, so that $\|$ is free. 
The following construction is similar to the Katětov extension found for example in [3] and [4].

Let $X$ be a topological space, and let $F$ be a set of open filters on $X$. Let $X_{F}$ be the disjoint union of $X$ and $F$. For each open $U$ in $X$, let $U^{*}=$ $U \cup\{\mathscr{F} \in F \mid U \in \mathscr{F}\}$. Note that $(U \cap V)^{*}=U^{*} \cap V^{*}$ for every open $U$ and $V$ in $X$, and $\phi^{*}=\varnothing$. Let $X_{F}$ have the topology generated by the base $\mathscr{B}=$ $\left\{U^{*} \mid U\right.$ is open in $\left.X\right\}$. Clearly $X$ is a dense subspace of $X_{F}$.

THEOREM 3. If $F$ is any of the following sets,

(a) all open filters on $X$,

(b) all open ultrafilters on $X$,

(c) all free open ultrafilters on $X$, then $X_{F}$ is a Baire space.

Proof. We shall prove Theorem 3 for case (c). Assume that $X_{F}$ is not a Baire space. Let $\left\{U_{i}\right\}$ and $\left\{B_{i}\right\}$ be defined as in the Lemma for the space $X_{F}$. Each $B_{i}=V_{i}^{*}$ for some open $V_{i}$ in $X$. Let $\mathscr{U}$ be an open ultrafilter on $X$ containing $\left\{V_{i}\right\}$. Since $\bigcap_{i=1}^{\infty} V_{i} \subset \bigcap_{i=1}^{\infty} B_{i} \subset \bigcap_{i=1}^{\infty} U_{i}$ and $\left\{U_{i}\right\}$ is point finite, then $\bigcap_{i=1}^{\infty} V_{i}=\varnothing$. Therefore $\cap \mathscr{U}=\varnothing$, so that $\mathscr{U}$ is free. Hence $\mathscr{U} \in F$. Since each $V_{i} \in \mathscr{U}$, then $\mathscr{U} \in V_{i}^{*}=B_{i}$. Thus $\mathscr{U} \in \bigcap_{i=1}^{\infty} B_{i} \subset \bigcap_{i=1}^{\infty} U_{i}=\varnothing$. This contradiction establishes that $X_{F}$ is a Baire space.

COROLlary. Every topological space is a dense subspace of some compact Baire space.

Proof. If $X$ is a topological space, let $X_{F}^{*}$ be the one-point compactification of $X_{F}$, where $F$ is any of the sets given in the statement of Theorem 3. Since $X_{F}$ is a dense open subspace of $X_{F}^{*}$, and $X_{F}$ is a Baire space, then $X_{F}^{*}$ must be a Baire space.

THEOREM 4. Let $F$ be the set of all free open ultrafilters on $X$, and let $f$ be a continuous function from $X$ into a $T_{3}$-space $Y$ such that $f(X)$ is dense in $Y$. Then there exists a subspace $Z$ of $Y_{F}$ containing $X$ and a continuous function $g$ from $Z$ onto $Y$ such that $\left.g\right|_{X}=f$ and $\left.g\right|_{Z \backslash X}$ is a homeomorphism from $Z \backslash X$ onto $Y \mid f(X)$. Furthermore, if $f$ is a homeomorphism then $g$ is a homeomorphism.

Proof. For each $y \in Y \backslash f(X)$, let $\mathscr{V}_{y}$ be the open filter on $Y$ consisting of all open subsets of $Y$ containing $y$. Let $\mathscr{U}_{y}$ be an open ultrafilter on $X$ generated by $f^{-1}\left(\mathscr{V}_{y}\right) \cdot \mathscr{U}_{y}$ is free since if $x \in X$, then there exists an open set $V$ in $Y$ with $y \in V$ and $f(x) \notin V$. Since $V \in \mathscr{V}_{y}, f^{-1}(V) \in \mathscr{U}_{y}$. But $x \notin f^{-1}(V)$, so that $\mathscr{U}_{y}$ must be free. Let $Z=X \bigcup\left\{\mathscr{U}_{y}|y \in Y| f(X)\right\}$. Define $g: Z \rightarrow Y$ by $g(x)=f(x)$ if $x \in X$ and $g\left(\mathscr{U}_{y}\right)=y$ for each $y \in Y \backslash f(X)$. To see that $g$ is well defined, let $y_{1}$ and $y_{2}$ be distinct points of $V \backslash f(X)$. Then there exists an open $U$ in $Y$ such that $y_{1} \in U$ and $y_{2} \notin \bar{U}$. Hence $U \in \mathscr{V}_{y_{1}}$ and $Y \backslash \bar{U} \in \mathscr{V}_{y_{2}}$. But then $f^{-1}(U) \in \mathscr{U}_{v_{1}}$ and $X \mid f^{-1}(\bar{U}) \in \mathscr{U}_{\nu_{2}}$. Therefore $\mathscr{U}_{v_{1}} \neq \mathscr{U}_{v_{2}}$. 
The fact that $g$ is continuous follows from the fact that $X$ is dense in $Z$, the fact that $Y$ is regular, and the fact that for each $z \in Z$ the restriction $g \mid X \bigcup\{z\}$ is continuous.

To see that $\left.g\right|_{Z \backslash X}$ is a homeomorphism, let $h=\left.g\right|_{Z \backslash X}$. Let $y \in Y \backslash X$, and let $U^{*}$ contain $h^{-1}(y)$ for some open $U$ in $X$. Then $h^{-1}(y)=\mathscr{U}_{y}$ and $U \in \mathscr{U}_{y}$. Hence $f^{-1}(V) \subset U$ for some $V \in \mathscr{V}_{y}$. Let $y^{\prime} \in V$. Then $V \in \mathscr{V}_{y^{\prime}}$, so that $f^{-1}(V) \in \mathscr{U}_{y^{\prime}}$. But then $U \in \mathscr{U}_{y^{\prime}}$, so that $h^{-1}\left(y^{\prime}\right)=\mathscr{U}_{y^{\prime}} \in U^{*}$. Therefore $h^{-1}(V) \subset U^{*}$.

Finally, suppose that $f$ is a homeomorphism. Let $y \in Y$, and let $U$ be open in $X$ such that $g^{-1}(y) \in U^{*}$. If $y \in f(X)$, then there exists an open $V$ in $Y$ such that $f^{-1}(V) \subset U$. If $y \in Y \mid f(X)$, then $g^{-1}(y)=\mathscr{U}_{y} \in U^{*}$, so that $U \in \mathscr{U}_{y}$. But then there exists an open subset $V$ of $Y$ containing $y$ such that $f^{-1}(V) \subset U$. In either case, let $y^{\prime} \in V$. If $y^{\prime} \in f(X)$, then $g^{-1}\left(y^{\prime}\right)=f^{-1}\left(y^{\prime}\right) \in f^{-1}(V) \subset U \subset U^{*}$. If $y^{\prime} \in Y \mid f(X)$, then $V \in \mathscr{V}_{y^{\prime}}$, so that $f^{-1}(V) \in \mathbb{l}_{y^{\prime}}$. Hence $U \in \mathscr{U}_{y^{\prime}}$, so that $g^{-1}\left(y^{\prime}\right)=\mathscr{U}_{y^{\prime}} \in U^{*}$. Therefore, since $y^{\prime}$ is arbitrary, $g^{-1}(V) \subset U^{*}$.

Corollary 1. If $X$ is a $T_{3}$-space, then every $T_{3}$-space extension of $X$ can be embedded as a subspace of $X_{F}$ containing $X$.

COROLlary 2. Let $X$ be a Tychonoff space and $Y$ be a Hausdorff space. Then $Y$ is a compactification of $X$ if and only if $Y$ can be embedded as $a$ compact subspace of $X_{F}$ containing $X$.

We might note that it follows from Corollary 2 that $X_{F}$ is in general not Hausdorff. In fact if $X$ is $H$-closed, no subset of $X_{F}$ containing $X$ as a proper subset is ever Hausdorff. This is because a Hausdorff space $X$ is $H$-closed if and only if every embedding of $X$ into a Hausdorff space is a closed embedding. Therefore if $X$ is an $H$-closed space which is not a Baire space (e.g., Herrlich's example in [2]), then $X$ is a Hausdorff space which cannot have a Hausdorff Baire space extension. Observe that $X$ could never be regular, since a regular $H$-closed space must be compact, and hence a Baire space. It would be of interest to know whether every regular space has a regular Baire space extension.

\section{REFERENCES}

1. J. R. Fogelgren and R. A. McCoy, Some topological properties defined by homeomorphism groups, Arch. Math. 9 (1971), 1-6.

2. H. Herrlich, Nicht alle $T_{2}$-minimalen Räume sind von 2. Kategorie, Math. Z. 91 (1966), 185. MR 32 \#6392.

3. M. Katětov, Über H-abgeschlossene und bikompakte Räume, Casopis Pěst. Mat. Fys. 69 (1940), 36-49. MR 1, 317.

4. C. T. Liu, Absolutely closed spaces, Trans. Amer. Math. Soc. 130 (1968), 86-104. MR 36 \#2107.

Department of Mathematics, V'irginia Polytechnic Institute and State UniVERSITY, BLACKSBURG, VIRGINIA 24061 